\section{A histological procedure to study fungal infection in the wax moth Galleria mellonella}

\author{
F. Perdoni, ${ }^{1}$ M. Falleni, ${ }^{1,2}$ D. Tosi, ${ }^{1,2}$ \\ D. Cirasola, ${ }^{1,3}$ S. Romagnoli, ${ }^{2}$ P. Braidotti, ${ }^{2}$ \\ E. Clementi, ${ }^{4}$ G. Bulfamante, ${ }^{1,2}$ E. Borghi ${ }^{1}$
}

'Department of Health Sciences, University of Milan

2Pathology Unit, San Paolo Hospital, Milan

${ }^{3}$ Specialization School in Microbiology and Virology, University of Milan

${ }^{4}$ Department of Biology and

Biotechnology “Lazzaro Spallanzani”, University of Pavia, Italy

\section{Abstract}

The invertebrate model Galleria mellonella is a widely used factitious host to study the microbial pathogenesis in vivo. However, a specific procedure for the recovery and the processing of the infected tissues, important for a better understanding of the host-pathogen interactions, has not been reported to our knowledge. In the present study we describe a new procedure of fixation and processing of larval tissue that allows studying the larval topographic anatomy and assessing the morphological changes due to the fungal infection. Lepidopteran larvae were infected with Candida albicans strains displaying various biofilm-forming abilities. The whole larvae were then examined for tissue changes by histological techniques. We show that comparing cutting planes, serial transversal sections of paraffin-embedded larva result in better accuracy and information recovering. Using this technique, it was possible to preserve the integrity of $G$. mellonella internal structures allowing the detailed analysis of morphological differences in different experimental groups (i.e., healthy $v s$ infected larvae). We were also able to study strain-related differences in the pathogenesis of $C$. albicans by observing the immune response elicited and the invasiveness of two isolates within the larval tissues.

In general, by processing the whole larva and optimizing routinely histochemical stainings, it is possible to visualize and analyse infected tissues. Various degrees of pathogenicity (strain- or inoculum-related), and the infection time course can be described in details. Moreover, the host immune response events can be followed throughout the infectious process leading to a comprehensive picture of the studied phenomenon.

\section{Introduction}

In the past few decades, there has been a growing public consciousness for novel experimental model hosts of human pathogens that are ethically more acceptable than whole mammalian models. However none of the invertebrate models adopted can be representative of all the pathogenic aspects of host-pathogen interactions. ${ }^{1}$ The choice of the most appropriate model is a critical step in the experimental design, depending on the pathogenic feature of interest. The lepidopteran Galleria mellonella is susceptible to infections by a large number of microorganisms also taxonomically unrelated as fungi and bacteria. This model has been previously successfully used as a tool to study the pathogenicity of yeast clinical isolates, having a similar immune response to mammals. ${ }^{2}$ The wax-moth larva model, compared to other invertebrates, offers some advantages such as the ability to grow at mammalian temperature (i.e., $37^{\circ} \mathrm{C}$ ) and the possibility to recover infected tissues. ${ }^{1}$ The latter is extremely important to further evaluate host responses, and tissue invasion by the pathogen.

Insects, though lacking adaptive immune response typical of vertebrates, possess welldeveloped innate responses including physical barriers, such as integument and gut, and, after this first line of defence, also host immune cells and antimicrobial molecules.-5 The immune system of insects exhibits a high degree of structural and functional similarity with the innate immune system of mammals. ${ }^{6}$ The antifungal immunity of insects to fungal pathogens involves cellular and humoral components. The hemolymph system of wax moth, which contains different types of hemocytes, plays a role in recognition and elimination of microorganisms. It is important to note that insects and humans share some evolutionary conserved families of antimicrobial peptides such as the defensins and specific inhibitors against virulence associated metalloproteinases. ${ }^{7,8}$ Most of the studies using the Galleria model for the understanding of microbial pathogenesis are focused on the screening of laboratory mutants by means of survival curves, in order to identify genes potentially relevant for the pathogen virulence. ${ }^{1,9}$ The recovery of infected tissue, when performed, often implies the squeeze of the whole fat-body tissue of the larva allowing the visualization of the pathogens, but with an important loss of the insect anatomy and morphology. ${ }^{10}$

The purpose of our study was to set up histological procedures to study the hostpathogen interaction in vivo, by optimizing common laboratory techniques of fixation, processing, and staining to the experimental
Correspondence: Dr. Elisa Borghi, Department of Health Sciences, University of Milan, via A. di Rudinì 8, 20142 Milan, Italy.

Tel. +39.02.50323287.

E-mail: elisa.borghi@unimi.it

Key words: Galleria mellonella, Candida albicans, host-pathogen interaction.

Acknowledgments: the authors would like to thank Erika Messina and Roberto Tironi for technical assistance, and Profs. Giulia Morace and Luciano Sacchi for critical reading of the manuscript. This work was partially sponsored by a grant to EB from the European Society of Clinical Microbiology and Infectious Disease (ESCMID RESEARCH GRANT 2013).

Received for publication: 3 June 2014.

Accepted for publication: 29 July 2014.

This work is licensed under a Creative Commons Attribution NonCommercial 3.0 License (CC BYNC 3.0).

(C) Copyright F. Perdoni et al., 2014

Licensee PAGEPress, Italy

European Journal of Histochemistry 2014; 58:2428 doi:10.4081/ejh.2014.2428

model Galleria mellonella. In the present study, we used two isolates of Candida albicans, the most common species causing invasive fungal infection ${ }^{11}$, differing in the in vitro biofilm-forming ability, and in the infected larvae survival. ${ }^{12}$ To describe the infectious process, the infected larvae were processed at three time points and sectioned, and several histological staining were adapted to the waxmoth larva tissues to highlight the insect response to the yeast invasion.

A comparison between a traditional fat-body squeeze and a whole larva processing was also carried out.

\section{Materials and Methods}

Final instar larvae of Galleria mellonella were purchased from the Allevamento Cirà, Como, Italy. Larvae were stored in wood shavings in the dark at $15^{\circ} \mathrm{C}$ prior to experiment. All larvae were used within one week from receipt. Two Candida albicans strains were used in the study; the reference strain SC5314, strong biofilm-producer, and a clinical strain CaBA22, biofilm non-producer. ${ }^{12}$ Overnight YPD cultures of each isolate were washed, counted by hemocytometer, and standardized to $1 \times 10^{7}$ cells/mL in PBS. G. mellonella larvae were injected with $10^{5} \mathrm{CFU} / \mathrm{larva}$, directly into the hemocoel via the last left proleg, using a Hamilton syringe, and incubated at $37^{\circ} \mathrm{C}$ in 
plastic Petri plates. ${ }^{9,12}$ Each experimental group contained 16-20 randomly chosen larvae of appropriate weight $(330 \pm 20 \mathrm{mg})$; all experiments included a control group consisting in an equal number of larvae injected with sterile PBS to monitor the trauma, and non-injected larvae. At 24,48 and $72 \mathrm{~h}$ post-infection, larvae were processed by two different methods: i) fat body collection (squeeze), and ii) whole larvae body collection. For each time point and group, at least six larvae were used.

\section{Fat body collection (squeeze)}

Infected larvae were cut from the upper part of the body, and gently pressed allowing the hemolymph to exit. Once the hemolymph has been extruded and discarded, the larva was held over a sterile tube containing $1 \mathrm{~mL}$ of $10 \%$ buffered formalin, and then squeezed to collect the fat body. The tube was stored at $4^{\circ} \mathrm{C}$ overnight to block melanization, and to allow fixation. Fixed tissue was processed by means of an automatic apparatus (Leica ASP3005, Wetzlar, Germany), and routinely paraffin embedded as previously described..$^{13}$ Briefly, processing was performed overnight by means of a serial steps where dehydrating reagents are injected into the station room following standard protocols. Sections were then harvested with a painting brush on Super Frost Plus (Thermo Scientific, Waltham, MA, USA) slides, and stained.

\section{Whole larvae processing}

Since the larval exoskeleton is resistant to known fixative reagents, we performed an adequate fixation by injecting buffered formalin $10 \%$ with an insulin syringe in the last left proleg. The volume of solution injected, to have a turgid consistency of the larvae, was about $100 \mu \mathrm{L}$. Larvae were then stored at $4^{\circ} \mathrm{C}$ for 24 $\mathrm{h}$, to fix internal organs and block melanization. Whole larvae were dissected transversally or sagittally into two halves by means of an anatomic pincers and by using a new lancet blade for each larva. The procedure was carefully performed to avoid the squeeze of the larval tissues. The two halves of larvae were placed in the same BioCassette and routinely processed in the path lab. For transversally sectioned larvae, each paraffin-embedded half was further sectioned into two/three rings, after cooling the larva at room temperature for a few minutes to harden the tissues. A crucial step to avoid the paraffin block rupture during microtome sectioning was to keep the cut larval tissues in hot paraffin for one hour to stabilize the inclusion, and to obtain a complete merge of the cut rings in the final paraffin block. Finally, four/six rings (one in the distal part, two in the middle, and one in the proximal part) were positioned in each paraffinblock. Histochemical staining on slides with serial tissue sections was then performed: haematoxylin and eosin (HE) was used to evaluate tissue morphology, periodic acid Schiff (PAS) and Grocott Methenamine staining (GMS) to highlight fungi localization and host interaction, Giemsa, Alcian blue at various $\mathrm{pH}$ $(1,2.5$, and 3.1$)$ to evaluate hemocytes, and Feulgen staining to evaluate DNA. All histochemical stainings were performed according to standard laboratory protocols. ${ }^{14}$ Sagittally sectioned larvae were routinely paraffinembedded..$^{13} \mathrm{~A}$ distance of $50 \mu \mathrm{m}$ was maintained between serial 4-micron-thick tissue sections of the two halves, and the slides were stained with haematoxylin and eosin.

The microscopic visualization has been performed using a Leica Microscope DMLB, and the image acquisition with the NanoZoomerXR C12000 series (Hamamatsu Photonics K.K., Tokio, Japan.).

\section{Results}

To optimize the study of host-pathogen interactions, two tissue collection techniques were compared: fat body squeeze and whole larva processing (Figure 1).

\section{Fat body collection (squeeze)}

The processing by squeezing the larval tissues allowed the visualization of the fat body cellular morphology, and of some intestinal and tracheal structures (Figure 1A). An overall good tissue larval morphology was achieved: fragments of the fat body sometimes entrapping muscle fibers and short tracts of tracheae, limited segments of the intestinal tube, and some hemocytes were observed in each sample.

\section{Whole larva processing}

The positioning of four to six different rings in a sole paraffin block facilitated the tridimensional reconstruction of the larval anatomy, and the study of the progression of the infec- tion into the tissues (Figure 1B). The evaluation of sagittal sections, obtained by cutting the entire paraffin-block, was used mainly for the assessment of the whole larva anatomy. A good morphology of the $G$. mellonella cuticle and internal organs was obtained coupled with a topographical reconstruction of its anatomy (Figure 1C).

\section{Host-fungus interaction}

We were able to follow the infectious process by both the used methods. In the squeezed tissues, the infectious agent, in its different morphological forms of yeast and hyphae, was visualized among the tissues, rarely incorporated in melanized nodules (Figure 2 A-D). The CaBA22 biofilm non-producing strain showed a slower progression of the infection (Figure 2A), with small nodules dispersed in the fat body. Blastocells were the most common morphological form detected in the larval tissues. The strain showed a tropism for the gut epithelium, but the lack of a strong filamentation resulted in the sequestering of the pathogen mainly outside the gut wall (Figure 2B). The SC5314 isolate, biofilm-producer, resulted in more rapid progression of infection characterized by the presence of simultaneous foci of fungus replication (Figure 2C). The strain showed a high propensity to filament and a massive invasion of the intestinal outer layer (Figure 2D). In the whole larval embedding (Figure $2 \mathrm{E}-\mathrm{L}$ ), the preservation of the cuticle allowed observing the nodules localization into the larva. The CaBA22 isolate was mainly encapsulated in small nodules localized preferentially in the subcuticular area (Figure 2 E,I,J). The CaBA22 was confirmed to be poorly able to filament, and showed a lesser capability to invade larval tissues (Figure 2F).

Differences in the capability of the two isolates to filament and to invade the larval organs were fully evident during the progression of the infection. As previously described by our group, ${ }^{15}$ the SC5314 isolate showed a

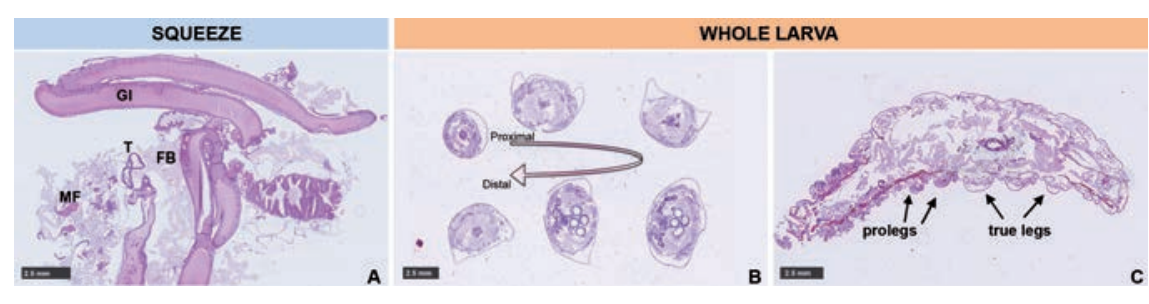

Figure 1. Comparison of the two methods used for larval tissues processing. Control larvae were processed in parallel, and stained with $\mathrm{HE}$. A) Fat body recovery by squeezing: segments of the gastrointestinal (GI) tract, tracheal (T) system, fat body (FB), and muscle fibers (MF) are visible in a section from squeezed paraffine-embedded material. $B, C$ ) Whole larva processing: B) proximal, middle and distal transversal sections are visible in a sole slide. C) Sagittal section of the larval body. 
strong tropism for the gut epithelium (Figure 2 $\mathrm{G}, \mathrm{H})$, with some fungal elements reaching the intestinal wall even in the first phase of the infection $(24 \mathrm{~h})$. The growth of the fungus and its filamentation led to midgut walls invasion at $48 \mathrm{~h}$ post infection (Figure $2 \mathrm{G}, \mathrm{H}$ ). The SC5314 isolate induced nodules with stronger melanization (Figure 2K), and with the tendency to converge in large aggregates (Figure $2 \mathrm{~K}, \mathrm{~L})$. The nodules were localized more deeply within the fat body, around the intestinal wall and, although more rarely, close to the tracheal system (Figure $2 \mathrm{~K}, \mathrm{~L}$ ). The Feulgen staining, initially performed to assess the possible presence of extracellular DNA in the fungal biofilm, was very useful to visualize the melanin distribution.

\section{Cellular immune response}

The whole larva processing allowed also the understanding of the time-dependent hemocytes recruitment in response to the $C$. albicans infection. Uninfected larvae, processed in the same way, were used for comparative purposes. Histochemical stainings were of great use in the hemocytes characterization: differences in shape, cytoplasmic staining affinity and inclusions were detected. Morphological aspects of host-pathogen interactions appeared particularly detailed: fungal pattern of growth at infections sites and the host organ damage were well depicted by PAS, GMS (not showed because it was overlapping with PAS results) and Feulgen stainings, whereas different hemocytes involved in response were well observed by Giemsa, and Alcian blue staining, giving information about the entity of infection and the virulence of pathogen.

Morphologically distinct immune cells were visualized in different tissues, and in nodules (Figure 3). Hemocytes localization in control larvae were mainly subcuticolar (Figure 3A), dispersed in the fat body (Figure 3B), and in discrete aggregates around intestinal and tracheal walls (Figure 3C). After the infection with both strains, in the first 24 hours, an increase of hemocytes was observed primarily at the inoculum site Figure 3D). During the infection progression (48 h), we observed, compared to control larvae, an increase number of immune cells in the fat body (Figure $3 \mathrm{E}$ ), and inside sparse small melanized nodules containing fungal elements. In the late phases of infection $(72 \mathrm{~h})$, and in relation to the pathogenicity degree of $C$. albicans isolates, the yeast reached and progressively surrounded the larval intestinal walls. A large number of hemocytes were then visualized next to intestinal and tracheal walls (Figure 3F). Cellular characterization of hemocytes, with different cytoplasmic granules, inclusions, and mucins production, were further visualized with the Alcian blue staining at $\mathrm{pH}$

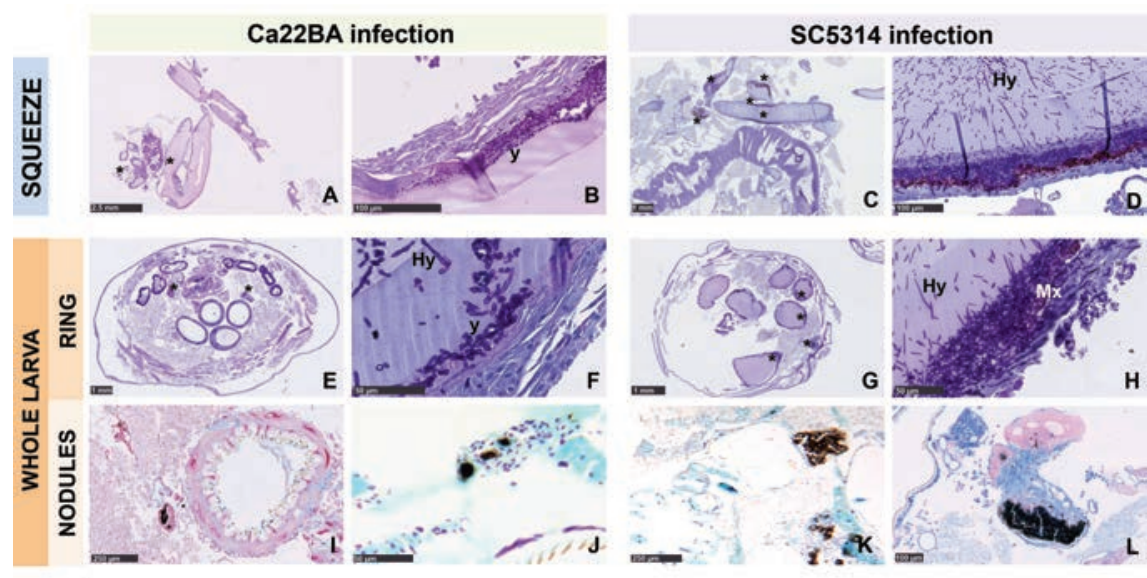

Figure 2. Host-pathogen interaction study by processing larval tissue by squeezing (A-D) and by whole larva analysis (E-L). A,B,E,F,I,J) Larvae infected with the biofilm non-producing $C$. albicans CaBA22 ( $48 \mathrm{~h}$ post-infection). The fungus showed a tropism for the intestinal tract; the presence of pseudohyphae is sporadic whereas the most common morphological form is the blastocells. F) The number of fungal elements able to reach the gut lumen is low and appreciable only at post-infection $72 \mathrm{~h}$. I,J) The nodules are smaller in dimension and usually isolated into the fat body. C, D, G, H, K, L) larva infected with the biofilm-producing $C$. albicans SC5314 (48 h post-infection): a high degree of fungal filamentation is demonstrated as well as the tendency to invade the gut wall (C, $\mathrm{D}, \mathrm{G}$, and $\mathrm{H}) . \mathrm{K}, \mathrm{L})$ The nodules are big in dimension and confluent. A-H) PAS staining. I) Alcian Blue pH 2.5. J,K) Feulgen. L) Giemsa. E,G) Middle sections of the larva. Hy, hyphae and pseudohyphae; Y, yeast cells; Mx, biofilm matrix; *, site of infection.
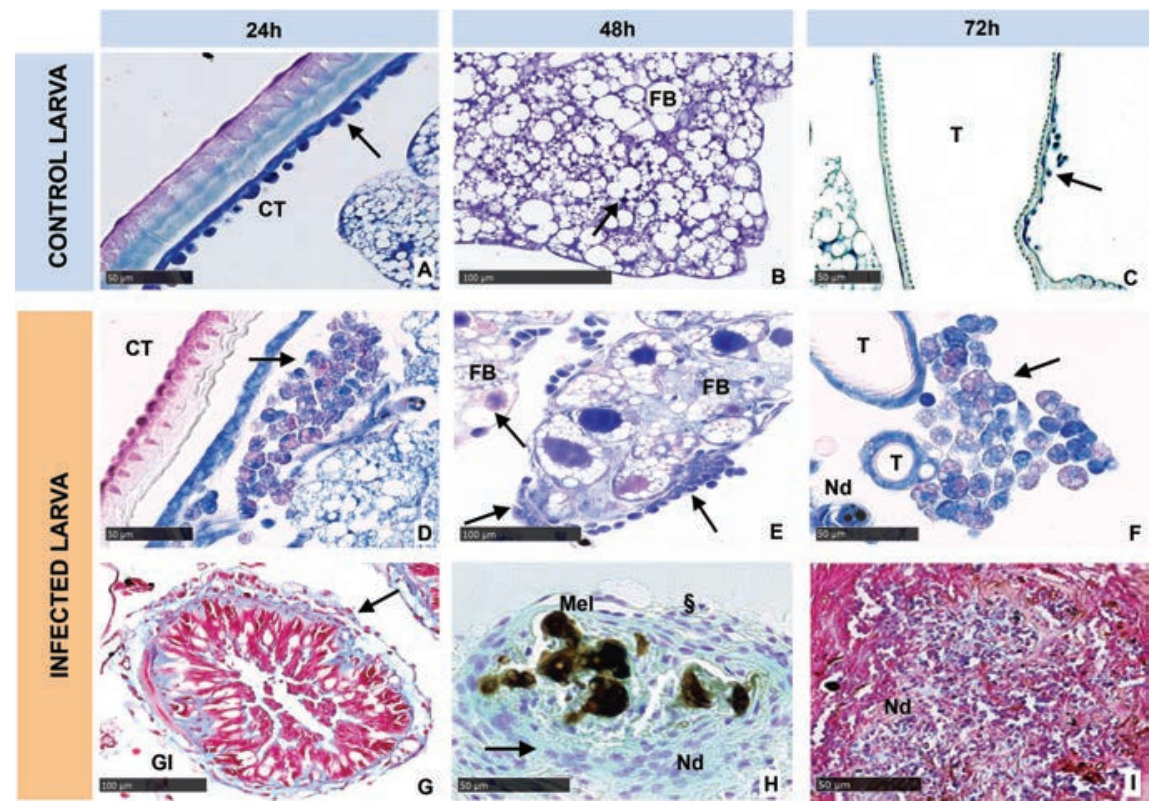

Figure 3. Cellular immune response in control larvae (A-C) and in infected larvae (D-I) in various phases of the infection $(24,48$, and $72 \mathrm{~h})$. A) Hemocytes monolayer localized sub-cuticularly (Giemsa staining). B) Scattered hemocytes in the normal fat body (PAS). C) small aggregates of hemocytes surrounding the tracheal walls in normal conditions (Feulgen). D) Hemocytes increase in the subcuticular area close to the injection area (Giemsa). E) Hemocytes increment in the fat body during the infection process (Giemsa). F) A small melanized nodule, and hemocytes recruitment close to tracheae in the later phases $(72 \mathrm{~h}$ ) of infection (Giemsa). G) Hemocytes aggregation and mucins characterization of a GI tract (Alcian blue $\mathrm{pH}$ 2.5). H) Melanine deposition in nodule with spindle cells clotting, and newer round hemocytes $(\$)$ recruited from the hemolymph (Feulgen). I) Mature nodule formed by hemocytes with different mucins-affinity acquisition (Alcian blue pH 3.1). Hemocytes are highlighted by arrows. CT, cuticle; FB, fat body; T, trachea; GI, gastro intestinal tract; Nd, nodule; Mel, melanin. 
2.5 (Figure $3 \mathrm{G}$ ) and Giemsa. Furthermore, different histochemical properties of hemocytes cellular membranes were appreciated particularly with Alcian blue $\mathrm{pH} 3.1$. Hemocytes recruitment at the infection site, and spindle cells forming the clots were observed in nodules entrapping fungal elements (Figure 3 $\mathrm{H}, \mathrm{I})$. Specifically, round hemocytes acquired a spindle shape to stratify around the infectious agent in the external part of melanized nodules, thus underlining their role in encapsulation (Figure $3 \mathrm{H}$ ).

\section{Discussion}

In vivo research addressing virulence of human pathogens is influenced by experimental costs, ethical concerns, and by the new European guiding principles ${ }^{16}$ focused on reducing, replacing, and refining the use of mammals as model hosts. Galleria mellonella is a widely adopted insect model to investigate the virulence of a broad range of human pathogens. $^{8-9,17}$ Its response to infections, based only on innate immune response, is considered a useful model for understanding the first steps of human host-pathogens interactions. ${ }^{7}$ In this work we described an optimization of the invertebrate model tissues analysis to better investigate microbial pathogenic mechanisms using standard laboratory techniques. Different conditions and experimental groups were analyzed and compared: $G$. mellonella larvae infected with Candida albicans strains and healthy controls were differently processed: fat body collection (squeeze) and whole larva embedding (sagittal and multiple transversal sections). Furthermore, to evaluate different morphological aspects of hostpathogen interactions, various histochemical stainings were used, in order to select the most informative ones.

Our studies documented that whole larva processing is the best method to investigate host-pathogen reactions and the virulence of an infecting agent. Even though both the methods can be easily and quickly performed in a pathology lab, with only minimal precautions to achieve an optimal fixation and inclusion, the whole larva processing gave more information concerning host immune response in relation to the pathogen virulence. Transversal and sagittal whole sectioning of larvae facilitated the topographical reconstruction of both external and internal organs. On the contrary, microscopic visualization of the squeezed material showed only segments of digestive and respiratory tracts, and some hemocytes interspersed in the fat body, failing to visualize the cuticle and subcuticular areas. The chronological aspects of host-pathogen interaction, immune cell maturation and migration at the infection sites, and the progressive organ damage, were also fully documented. On slides obtained by processing the larvae at different time points of infection (24 to $72 \mathrm{~h}$ ), the fungus (yeast cells or hyphae) was easily monitored according to the different strain-related behavior. The filamentation of the biofilm-forming strain allowed the fungus to invade progressively deep tissues of the insect. ${ }^{18}$ Differences in fungal filamentation and biofilm matrix, detected as amorphous weak eosinophilic interhyphal material, were evident, with filamentous fungi showing a more aggressive invasion of host digestive walls within a shorter time.

We were able to draw a comprehensive evaluation of hemocytes recruitment, and their dynamic organization into the hemolymph and other tissues during the immune cell response to fungal infection. Specifically, we could observe larva immune cells tropism at different sites of infection, their aggregation in nodules and tendency to confluence, which appeared strictly related to the different behavior of the $C$. albicans isolate used. This gave precious information about possible sites of hemocytes production, mobilization, and recruitment at infectious sites. In line with previous observations, ${ }^{7,19}$ we observed few morphologically distinct immune cells in healthy $G$. mellonella larvae. Up to date, different types of circulating hemocytes comprising plasmatocytes, granulocytes, spherulocytes, oenocytes, and adipohemocytes have been characterized in the insect hemoplymph. These cells are believed to origin from an immature prohemocyte and are known to be involved in phagocytosis, nodule formation and encapsulation during the immune response. ${ }^{7,17}$ The characterization of these blood cells is up to now mainly based on their morphology, but it could represent an oversimplification. A better classification based on molecular markers on their cell surface will improve our knowledge on hemocytes maturation and immune recruitment. This aspect is of particular relevance for tissue hemocytes.

In healthy larvae, we observed the presence of a small number of hemocytes in the subcuticular area, in the hemolymph, and in close association with aero-digestive tracts. This localization displayed an anatomical distribution that peculiarly reminds the human BALT (bronchial associated lymphoid tissue) and MALT (mucous associated lymphoid tissue) systems. After the initial phases of infection, we found the number of hemocytes to increase in the previous-mentioned sites, being the immune cells attracted where fungi proliferated and began to invade, whereas the circulating hemocytes were reduced. In line with previous reports, hemocytes were documented to be involved in clotting and formation of nodules with encapsulation at different infectious sites. ${ }^{7}$ Indeed, the hypothesis of a multistep maturation process during host immune response was supported; further studies with histochemical stainings are needed to better characterize morpho-functional aspects of hemocytes. Far away from areas where the infectious agent could be seen, hemocytes appeared as round or oval cells of various dimension, sometimes resembling small immature cells, with a variable number and type of cytoplasmic granules and vacuoles. In infection sites, hemocytes formed multicellular aggregates, i.e., nodules, to entrap the invading yeast. We observed the morphological switch from round hemocytes into spindle cells (Figure $3 \mathrm{H}$ ) that participate in the encapsulation process. During the infection progression, nodules increased in number and in dimension, depending on biological characteristics of the $C$. albicans strain. Encapsulation takes place in cases where the invading organism is too big to be phagocytized, and involves covering the parasite with multiple layers of hemocytes and/or a melanin coat. This feature was highly noticeable in larvae infected with SC5314, where the pronounced filamentation resulted in large hyphae aggregates that make the phagocytosis worthless.

All the above-described aspects were noticeable in both the sagittal and transversal sections of the whole larva processing methods, which are easily achievable in a routine pathology laboratory. However, transversal processing is less time- and money-consuming, allowing the visualization in a single slide of multiple anatomical levels. The $G$. mellonella model could support several fungal infections such as Cryptococcus spp. ${ }^{20}$ and Aspergillus spp., ${ }^{9,21}$ and a variety of different environmental and experimental conditions. Moreover, it allows choosing the route of infection (systemic injection, oral inoculation, or topical application) more representative for the infectious disease studied.

Given the relevance of the $G$. mellonella model to investigate human infections, the standardization of procedures for handling this model need to be implemented. The described technique adds crucial hints to its use for tissue recovering and analysis of hostpathogen interaction.

\section{References}

1. Desalermos A, Fuchs BB, Mylonakis E. Selecting an invertebrate model host for the study of fungal pathogenesis. PLoS Pathog 2012;8:e1002451.

2. Brennan M, Thomas DY, Whiteway M, 
Kavanagh K. Correlation between virulence of Candida albicans mutants in mice and Galleria mellonella larvae. FEMS Immunol Med Microbiol 2002;34:153-7.

3. Jiravanichpaisal P, Lee BL, Söderhäll K. Cell-mediated immunity in arthropods: hematopoiesis, coagulation, melanizationand opsonisation. Immunobiology 2006;211:213-36.

4. Lavine MD, Strand MR. Insect hemocytes and their role in immunity. Insect Biochem Mol Biol 2002;32:1295-309.

5. Scully LR and Bidochka MJ. Developing insect models for the study of current and emerging human pathogens. FEMS Microbiol Lett 2006;63:1-9.

6. Browne N, Heelan M, Kavanagh K. An analysis of the structural and functional similarities of insect hemocytes and mammalian phagocytes. Virulence. 2013;4:597-603.

7. Kavanagh K, Reeves EP. Exploiting the potential of insects for in vivo pathogenicity testing of microbial pathogens. FEMS Microbiol Rev 2004; 28:101-12.

8. Vilcinskas A. Coevolution between pathogen-derived proteinases and proteinase inhibitors of host insects. Virulence 2010;1:206-14.

9. Slater JL, Gregson L, Denning DW, Warn
PA. Pathogenicity of Aspergillus fumigatus mutants assessed in Galleria mellonella matches that in mice. Med Mycol 2011;49:S107-13.

10. Fuchs BB, O'Brien E, Khoury JB, Mylonakis E. Methods for using Galleria mellonella as a model host to study fungal pathogenesis. Virulence 2010;1:475-82.

11. Morace G, Borghi E, Iatta R, Amato G, Andreoni S, Brigante G, et al. Antifungal susceptibility of invasive yeast isolates in Italy: the GISIA3 study in critically ill patients. BMC Infect Dis 2011;11:130.

12. Cirasola D, Sciota R, Vizzini L, Ricucci V, Morace G, and Borghi E. Experimental biofilm-related Candida infections. Future Microbiol 2013;8:799-805.

13. Vacca LL. Laboratory manual of histochemistry. Raven Press, 1985.

14. Luna LG. Manual of histologic staining methods of the Armed Forces Institute of Pathology. McGraw-Hill Book Company, 1970.

15. Borghi E, Romagnoli S, Fuchs BB, Cirasola D, Perdoni F, Tosi D, et al. Correlation between Candida albicans biofilm formation and invasion of the invertebrate host Galleria mellonella. Future Microbiol 2014;9:163-73.
16. European Union. Directive 2010/63/EU of the European Parliament and of the Council of 22 September 2010 on the protection of animals used for scientific purposes. Retrieved: 12 August 2013. Available from: http://eur-lex.europa.eu/ LexUriServ/LexUriServ.do?uri=0J:L:2010: 276:0033:0079:en:PDF

17. Mizerska-Dudka M, Andrejko M. Galleria mellonella hemocytes destruction after infection with Pseudomonas aeruginosa. J Basic Microbiol 2014;54:232-46.

18. Mayer FL, Wilson D, Hube B. Candida albicans pathogenicity mechanisms. Virulence 2013;4:119-28.

19. Ribeiro C, Brehélin M. Insect haemocytes: what type of cell is that? J Insect Physiol 2006;52:417-29.

20. Gomez-Lopez A, Forastiero A, CendejasBueno E, Gregson L, Mellado E, Howard SJ, et al. An invertebrate model to evaluate virulence in Aspergillus fumigatus: the role of azole resistance. Med Mycol 2014; 52:311-9

21. Mylonakis E, Moreno R, El Khoury JB, Idnurm A, Heitman J, Calderwood SB, et al. Galleria mellonella as a model system to study Cryptococcus neoformans pathogenesis. Infect Immun 2005;73:3842-50. 